\title{
La valoración de empresas aplicada en las Mipymes de confecciones textiles de Cúcuta
}

Recibido:

Octubre 30 de 2012

Aceptado:

Marzo 12 de 2013

\footnotetext{
${ }^{1}$ M.Sc Gerencia de Empresas Mención Finanzas (Universidad Nacional Experimental del

Estado Táchira - Venezuela) M.Sc en Práctica Pedagógica (Universidad Francisco de Paula Santander - Colombia) Profesor adscrito del Programa de Contaduría Pública Universidad Francisco de Paula Santander, Cúcuta - Colombia. Grupo de Investigación CINERA. Correo electrónico:

gersonruedavera@ufps.edu.co
}

${ }^{2}$ M.Sc Gerencia de Empresas Mención Finanzas.

Director de Departamento de Ingeniería Mecánica,

Mecatrónica e Industrial -

Universidad de Pamplona

(Colombia).

Correo electrónico:

saurythomas@unipamplona. edu.co

Docente de Catedra Universidad Francisco de Paula Santander, Cúcuta - Colombia.

Grupo de Investigación CINERA.

Candidato a Magister en Planificación Global.

Universidad Pedagogica

Experimental Libertador UPEL Venezuela. Correo electrónico: yair2004_74@hotmail.com

\section{Resumen}

El propósito que planteó la investigación, fue determinar el conocimiento sobre valoración de empresa aplicado por parte de las Mipymes del sector textil y confecciones en el municipio de San José de Cúcuta (Norte de Santander - Colombia). Para lo cual se utilizó el tipo de investigación descriptiva; se aplicó una encuestaa las empresas del sector, teniendo en cuenta la teoría del valor, el ebitda(Beneficio antes de intereses, impuestos, depreciaciones y amortizaciones), el precio arbitraje, la valuación de valores, los residuos de los dividendos y la estructura del capital, para ejercer una comparación con la realidad. Para ello se recomienda tener en cuenta las tendencias del mercado, innovar procesos tecnológicos, aplicar algún método de valoración de forma constante e incrementar la capacidad de decisión.

Palabras clave: Valoración de empresas, textiles y confecciones, Mipymes.

\section{Abstract}

The intention that raised the investigation, was the knowledge determined on valuation of company applied on the part of the Mipymes textile sector and confections in the municipality of Cúcuta's San Jose (North of Santander Colombia). For which there was in use the type of descriptive investigation; a survey was applied to the companies of the sector, having in the price counts the theory of the value, the ebitda (benefit before interests, taxes, depreciations and amortizations), arbitration, the appraisal of values, the residues of the dividends and the structure of the capital, to exercise a comparison with the reality. For it one recommends to bear the trends of the market in mind, to introduce technological processes, to apply some method of valuation of constant form and to increase the capacity

Keywords: Company of valuation, textiles and garments, Mipymes. 
No. 1

\section{Introducción}

L

a realidad de la organizaciones en

la actualidad exige que recuperen inversión en el tiempo, Lawrence (1990) afirma que en esta búsqueda se deben maximizar las riquezas de los propietarios, donde la preocupación real va desde la venta de acciones, capitalizaciones, restructuraciones e incluso hasta la venta total de la empresa; si se habla de valoración de empresas, se puede afirmar que es la encargada de integrar y relacionar todas las actividades de una organización; por otra parte Escalera y Herrera(2009) opinan que al avanzar la globalización junto con el reto de cumplir los requisitos de calidad, hacen mayor el desafío competitivo para las pequeñas y medianas empresas ya que se deben adoptar nuevos esquemas y formas de conducir la organización, además de ello se requiere adoptar un pensamiento orientado a crear valor.

Desde el escenario nacional, Córdoba (2009) hace referencia a que la competencia en el mercado interno cada vez tiende a ser mayor y se refleja en la llegada y expansión de grandes cadenas internacionales donde las importaciones de confecciones crecieron en el año 2008 casi en 30\%. Además desde lo departamental, también se puede mencionar según datos del Banco de la república (2006) que el clúster de las confecciones es uno de los más importantes, registrando en casi todas sus etapas de producción una tendencia reciente a generar un mayor valor agregado en las prendas textiles por el trabajo manual de los acabados y la creatividad de los diseños, determinando así que la ciudad desde hace muchos años viene dedicándose a lasconfecciones textiles, incentivando a que se creen cada vez más empresas y fortaleciendo el crecimiento de la economía, con lo que se obtiene estabilidad y permanencia en el mercado.

\section{Antecedentes de la investigación}

Según estudio realizado en la Universidad de Chile por Salinas (2006) en la actualidad la creación de valor es uno de los principales objetivos de las empresas lucrativas, involucra la toma de decisiones financieras; es decir, ninguna organización puede pretender que la generación de valor no constituya un factor determinante e indispensable en la economía, al contrario todas las organizaciones que no adopten una gerencia basada en el valor están destinadas a la desaparición y quiebra total.

Con respecto a un análisis realizado en la Universidad Autónoma de San Luis de Potosí, Escalera y Herrera(2009) dicen: que desde la perspectiva del dueño y/o administrador, las decisiones financieras son determinantes para crear o destruir valor para la empresa, si bien se ha cuestionado que por sus características propias, las Mipymes no pueden medir el valor económico agregado, pero si es factible que se pueda investigar el desarrollo y evaluación de estrategias que promuevan el valor y le permitan ser competitiva.

De acuerdo con el estudio planteado por la Universidad CES - Unirosario, Granaday Correa (2010) afirman que el gerente financiero, es el responsable de medir la importancia de las variables individuales ya que exclusivamente de él dependen las decisiones que se tomen dentro de la organización, igualmente es necesario que el líder se encuentre constantemente en la determinación de factores que contribuyan a generar valor económico, también se señala que la falta de un mercado activo de empresas no cotizadas, afecta al proceso de valoración.

\section{Referentes teóricos}

Teoría del valor. Con respecto al tema Dillard (1966) puntualiza que: "Un sistema de economía en el que se omitiera el valor, se- 
ría como una lógica sin concepto, una ética sin deber, una estética sin expresión". De lo anterior se puede afirmar que el conocimiento del valor se convierte en una herramienta fundamental en la economía. El Ebitda (Earnings Before Interest, Taxes, Depreciaton, and Amortization)Según lo expuesto por Bastidas (2007) se considera como una medida de rentabilidad y por tanto es un indicador que permite aproximarse al valor de una empresa. Se puede afirmar que este indicador permite tener sólo una aproximación de agregación de valor, se debe actuar con cautela cuando se quiera utilizar este indicador ya que no en todos los casos posee exactitud; además, con lo expuesto por López y Luna (2001) es necesario tener claridad sobre la información que se quiere y por ello se debe sistematizar el proceso aplicando preguntas orientadoras como: valorar para que, valorar para quién y valorar en qué circunstancias.

Teoría del precio arbitraje. Acorde a esta teoría, Van Horne(1997) afirma que la teoría de precios de arbitraje (APT) es un modelo de equilibrio referente a la forma en que se determinan los precios de los valores. Desarrollada originalmente por Stephen A. Ross, esta teoría se basa en la idea de que en los mercados financieros competitivos el arbitraje asegurará que los activos sin riesgos proporcionen el mismo rendimiento esperado; el arbitraje simplemente significa encontrar dos cosas que sean la misma en esencia, y comprar la más barata y vender, o vender en corto tiempo la más cara.

Teoría de la valuación de valores. De acuerdo a Philippatos(1979) la teoría de la valuación del precio de las acciones se funda en el supuesto de que existen ciertas variables y relaciones significativas que son posibles abstraer y aplicar en un modelo de valuación. Muchas de estas variables las podemos cuantificar y emplear en modelos matemáticos de valuación de acciones, mientras que otras son simplemente cualitativas, útiles en modelo de valuación descriptivos.
Teoría residual de los dividendos. Según Serrano y Villareal (1988) las elaboraciones teóricas existentes sobre el tipo de política de dividendos más adecuada para una empresa son múltiples, la mayoría de ellas relacionan una política específica con el valor de la empresa donde el costo de financiamiento a través de utilidades retenidas es inferior al costo de financiamiento a través de emisiones de acciones; como consecuencia de la inexistencia de gastos de emisión que afectan negativamente el costo del financiamiento mediante la emisión de acciones. Por ello, se debería utilizar la mayor cantidad de utilidades retenidas en la configuración del componente patrimonial en la estructura óptima de capital, donde se considera lo propuesto por Gamboa (2003) respecto a que se debería retener la mayor cantidad posible de utilidades hasta constituir la componente patrimonial en la estructura óptima de capital, teniendo en cuenta los elementos a) Capital Invertido b) Rentabilidad del capital invertido c) El costo de financiamiento.

Teoría de la estructura de capital. Según Gallagher y Andew (2001) un interrogante vital en finanzas es que tanta deuda y capital contable debe tener una compañía en su estructura de capital. La estructura de capital corresponde a la combinación de fuentes de fondos que utiliza una empresa; además el volumen de apalancamiento operativo que tiene una compañía está determinado en gran medida por el capital contable original de los inversionistas, quienes deciden qué tipo de negocio se debe operar; adicionalmente establece que se debe tener en cuenta las tasa de interés ya que es un fenómeno monetario donde se considera que es una recompensa por esperar el retorno de la inversión.

\section{Referentes metodológicos}

El tipo de investigación aplicado según Méndez (2001) es cuantitativo no experimen- 
No. 1 tal, debido a que no se ejerció control sobre las variables.

El diseño de investigación aplicado según Hernández, Fernández y Baptista (2008)fue el transaccional descriptivo debido a que la investigación se basó en conocer el grado de valoración que poseen las empresas en el sector textiles y confecciones de la ciudad de Cúcuta. La población está determina por sus características definitorias, donde concuerdan sus características; para el estudio la población estuvo compuesta por 546 empresas registradas en la cámara de comercio de Cúcuta.

Según Namakforoosh(2006) afirma que el tipo de muestra intencional obedece a la necesidad del investigador, donde los elementos muéstrales serán seleccionados bajo estricto juicio personal; para el desarrollo de la investigación se tomaron 50 empresas para conformar la muestra.

\section{Resultados y discusión}

A continuación se presenta el análisis y discusión de los resultados de la investigación con el propósito de establecer una comprensión de la realidad.

Figura 1. Considera que en los últimos tres años su empresa a generado valor.
Figura 2. Aplica estrategias para generar valor.

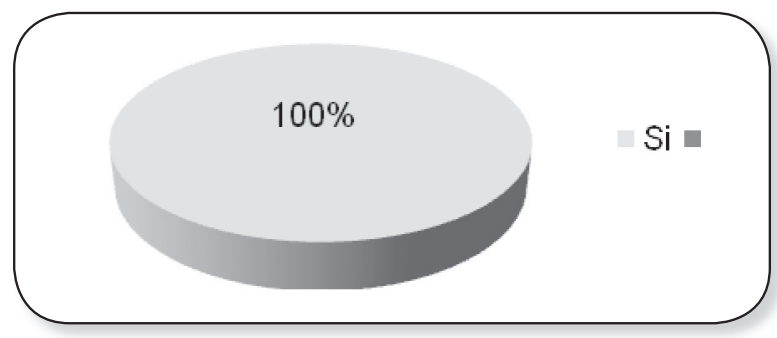

En respuesta del 100\% de los empresarios, todos aplican estrategias para generar valor.

Como la totalidad respondió que aplicaban estrategias se les pidió que respondieran que tipos de estrategias aplican para generar valor en su empresa y se presenta a continuación:

Figura 3. Estrategias aplicadas por los empresarios

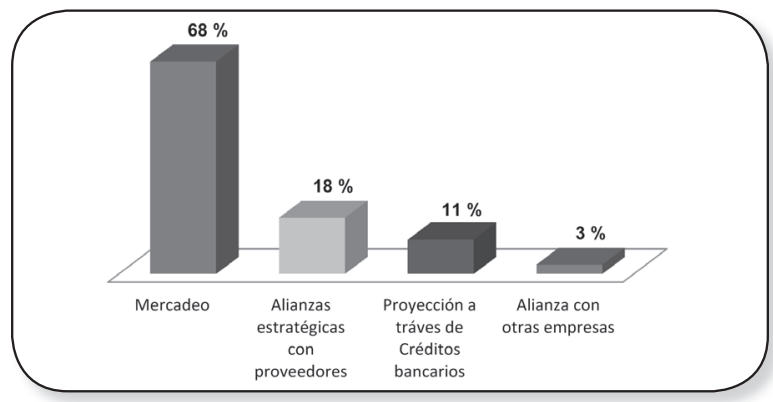

Se observa en la Figura 3 que la estrategia con mayor uso es el mercadeo, seguida de las alianzas con empresas que proveen al ente económico, esto significa que su mayor preocupación es incrementar los ingresos para ganar valor en el tiempo.

Figura 4. Periodos de Capacitación

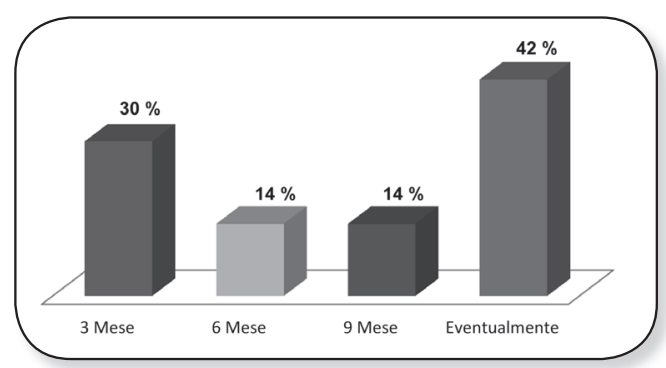

La capacitación de forma eventual de acuerdo a la Figura 4, es la más utilizada por las empresas, dejando así una sensación que los líderes de las organizaciones no la asumen como un elemento generador de valor empresarial, es por ello que se puede incurrir 
en errores de procedimiento que al final estén en contra de la valoración de la organización.

Figura 5. La reforma Física como generador de valor

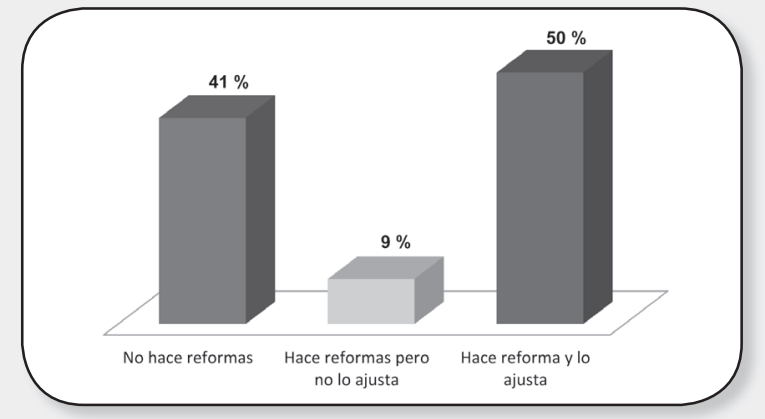

En la Figura 5 se puede observar que la mayoría de empresarios dicen ajustar las reformas físicas con el valor real de su empresa, con el animo de valorarla o que demuestre su inversión, pero por otra parte existe un número considerable de empresarios que afirman que no realizan reformas físicas en la empresa, es decir no se reconoce como un generador de valor para la organización.

Figura 6. Vincula profesionales para mejorar la calidad de sus productos.

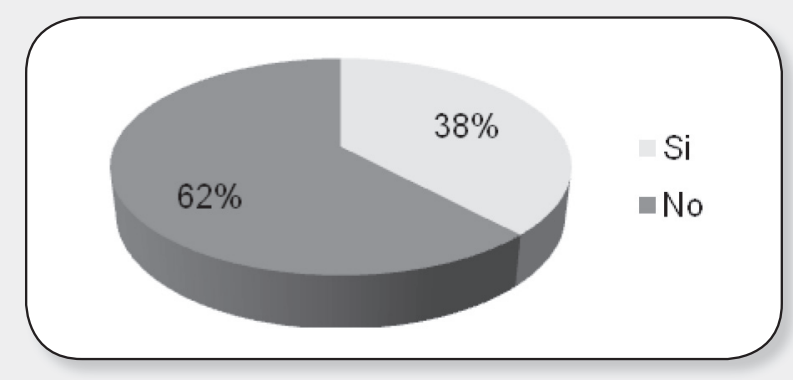

Acorde con la respuesta obtenida de los empresarios, se observa que vincular profesionales con el ánimo de aumentar la calidad de los productos no se ha tomado por parte de ellos como un generador de valor, olvidando que los procesos de calidad dentro de una empresa permitente que como ente económico mejore su valor (Figura 6).

De acuerdo al método de valoración de empresa basado en el balance, el 90\% de los empresarios considera que el valor de su empresa concuerda con el valor de su patrimonio extraído de los libros contables, lo que genera una reacción positiva con respecto a la transparencia de su inversión y que se espera una retribución o retorno que produzca un mayor valor (Figura 7).

Figura 7. Considera que el valor de la empresa concuerda con el valor de patrimonio en libros.

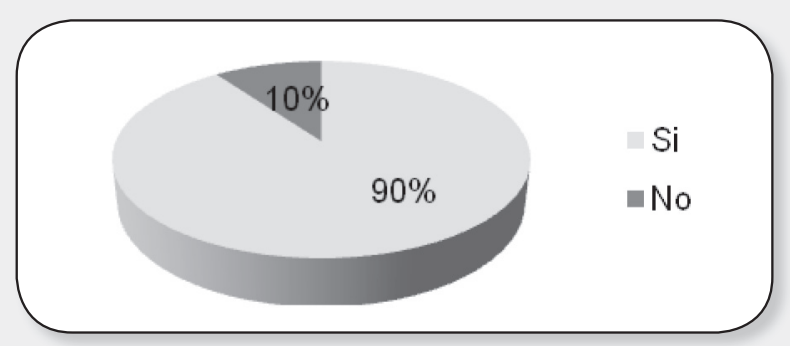

Adicionalmente se obtuvo la siguiente información:

De los empresarios encuestados el 70\% no ha realizado estudios de formación empresarial, el $98 \%$ de los empresarios conocen el costo de elaborar sus productos, el 60\% dice que en los últimos dos años el efectivo a influido de forma positiva, un 76\% afirma que los activos destinados a la producción maximiza los volúmenes de producción, el 68\% considera que el valor de su empresa se incrementa al realizar pago a los acreedores, cobro a los deudores, venta de los activos y retiro o aportes de las cantidades pendientes por los dueños de la empresa.

En respuesta de los encuestados el 68\% están en desacuerdo que el valor de su empresa se incrementa explicando que el verdadero valor se obtiene de sumar la compra de todos los activos nuevos, independientemente de la financiación tomando en cuenta la variante de valor por reposición, el $72 \%$ de los empresarios consideran que el valor de su empresa se obtiene de realizar un ajuste al valor de sus activos y pasivos, 100\% consideran que los métodos de valoración si impulsan el crecimiento de las micro, pequeñas y medianas empresas, el 100\% de los empresarios los cuales poseen su organización, afirman que les gustaría aplicar cualquier método de valoración de empresa el cual conlleve a mejorar sus flujos de efectivo.

Se tiene que el $50 \%$ de los empresarios es-
Enero-Junio 2013 ISSN 0122-820X 
No. 1

Enero-Junio 2013 ISSN 0122-820X timula el talento humano con bonificación y comisiones, un 34\% no otorgan ningún incentivo, el $16 \%$ solo en días especiales, respecto de la retroalimentación con los clientes, el $56 \%$ es a través de exhibiciones, 38\% línea de atención al cliente y $6 \%$ el buzón de sugerencias, para medir el desempeño de la empresa el $48 \%$ lo hace de forma detallada, el 32\% de forma global y el 20\% a través de alcance de objetivos, el $100 \%$ respondió que tienen en cuenta las tendencias para crear nuevos productos que generen valor en la empresa.

\section{Conclusiones y recomendaciones}

En cuanto a la valoración de empresas, se podría decir que genera preocupación en los empresarios por querer impulsar su formación empresarial, por otra parte, se debe establecer nuevas estrategias para sostenerse en el mercado, sin olvidar apropiarse con mayor rigurosidad del conocimiento de los costos de la producción, adicionalmente se puede adelantar políticas para estimular el talento humano, para así buscar en forma continua lograr la satisfacción de los clientes tanto interno como externo, con el fin de obtener una mejor retroalimentación por parte de cada uno de ellos.

Para emprender estrategias que ayuden a generar una corriente de beneficios para las empresas en un largo plazo, se deben determinar a través de la aplicación de cualquiera de los métodos de valoración de empresas, sumándole proyecciones con créditos blandos y en últimas la alianza con otras empresas, con el firme propósito de lograr alcanzar nuevas formas de incrementar valor y mantenerse a la vanguardia de los mercados.

Se recomienda descubrir nuevos segmentos de mercado, identificando los sectores que son desatendidos e insatisfechos, por otro lado es necesario canalizar nuevas ideas al trabajo para mejorar los procesos y obtener mejores resultados, de manera tal que las empresas puedan aplicar diversas estrategias para incrementar su valor en el largo plazo, posteriormente aplicar un sistema de costeo que pueda mejorar el control y le proporcione información, sin embargo esto depende fundamentalmente de la capacidad de decisión y empuje del empresario para implementar cambios.

\section{Agradecimientos}

Daxy Gisselth Fernández Peña, por su participación en el desarrollo de la investigación.

\section{Referencias}

Bastidas, C. (2007) Ebitda, ies un indicador financiero contable de agregación de valor? Revista CapivReview. Chile. Vol. 5. ISSN 0718-4654.

Banco de la Republica. (2006) Panorama económico regional. Boletín Económico Regional, Región Centro-Oriente. $\mathrm{N}^{\circ} 2$ mes de diciembre.

Córdoba, R. (2009) Los indicadores de los sectores de talla mundial. Revista dinero. $\mathrm{N}^{\circ} 326$ mes de mayo. ISSN 0122-1531.

Dillard, D. (1966) El fondo clásico. La teoría económica. Editorial Aguilar. Madrid. España.

Escalera, M. y Herrera, G. (2009) Modelo para generar valor económico agregado para las empresas. Revista Contribuciones a la economía. $\mathrm{N}^{\circ} 7$ mes de enero. ISSN 16368360 .

Gallagher, T y Andrew, J. (2001) Administración financiera, teoría y práctica. 2 Edición. Editorial Pearson. Colombia

Gamboa, J. (2003) Finanzas para sobrevivir: Un enfoque para la pequeña y mediana empresa. Editorial Alfaomega. Colombia

Granada, L. y Correa, L. (2010) Propuesta metodológica de valoración de empresas bajo el 
modelo de flujos de caja descontados, viabilidad financiera aplicada a la ESE "BelloSalud". Universidad CES - UNIROSARIO. Facultad de Medicina. Tesis de Maestría en Administración de Salud.

Hernández, R; Fernández, C. y Baptista, P. (2008) Metodología de la investigación. 3 Edición. Editorial McGraw-Hill. México.

Lawrence, G. (1990) Fundamentos de administración financiera. Editorial Harla S.A. México.

López, F. y Luna, W. (2001) Valoración de empresas en la práctica. Editorial McGraw-Hill. España.

Méndez, C. (2001) Metodología: diseño y desarrollo del proceso de investigación. 3 Edición. Editorial McGraw-Hill. Colombia.

Namakforoosh, M. (2006) Metodología de la investigación. 2 Edición. Editorial Limusa. México.

Philippatos, G. (1979) Fundamentos de administración financiera, texto y casos. Editorial McGraw-Hill. México.

Salinas, P. (2006) Valoración de empresas a través del método de comparables: Evidencia Chile. Universidad de Chile. Escuela de Economía y Administración. Tesis de pregrado no publicada.

Serrano, J.y Villareal, J. (1988) Fundamentos de finanzas. Editorial McGraw-Hill. Colombia.

Van Horne, C. (1997) Administración financiera. 10 Edición. Editorial Prentice Hall. México. 